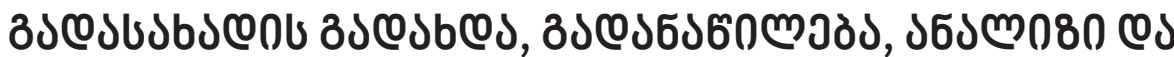

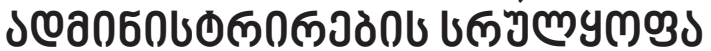

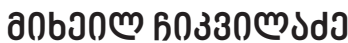

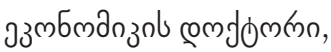

https://doi.org/10.35945/gb.2017.04.007

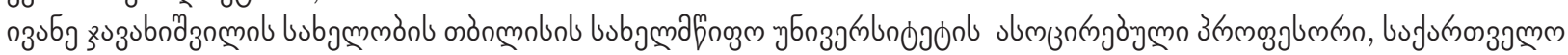

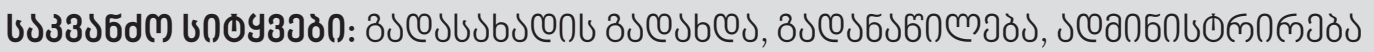

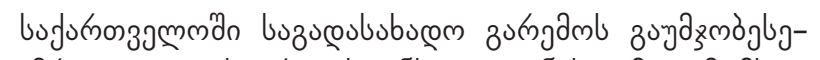

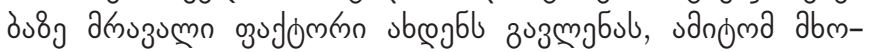

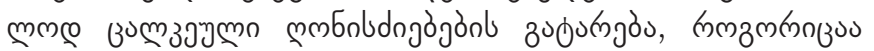

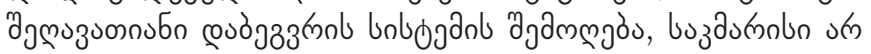

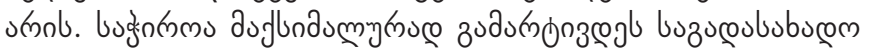

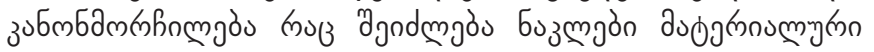

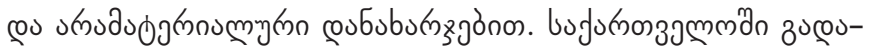

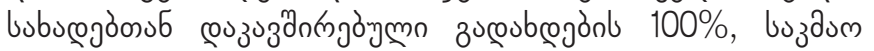

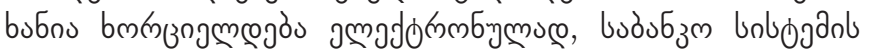

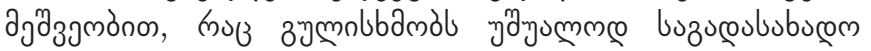

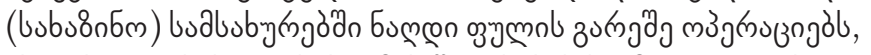

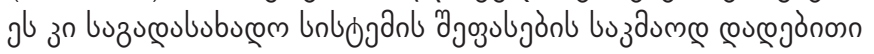

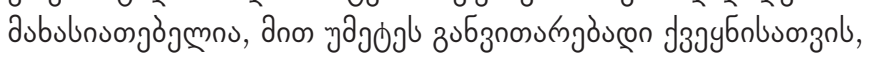
аБпдз

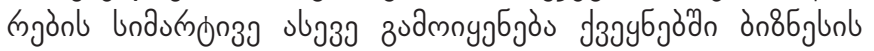

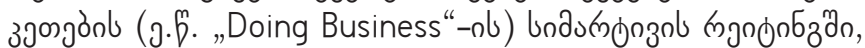

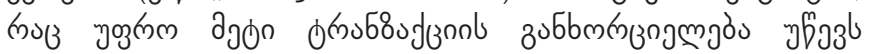

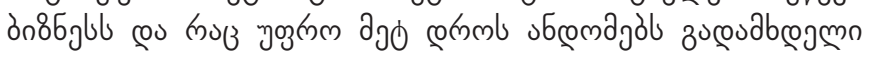

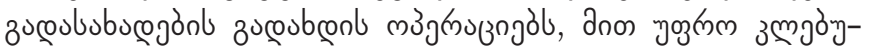

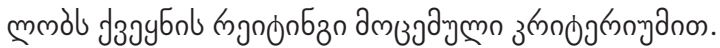

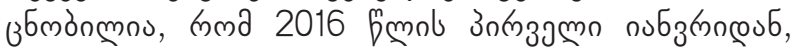

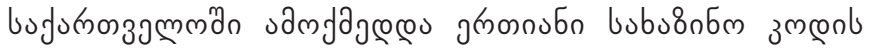

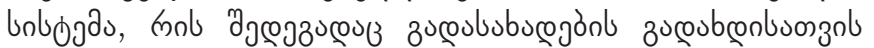

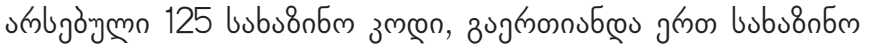

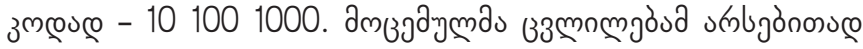

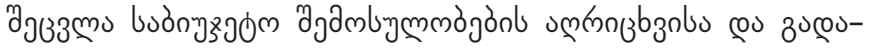

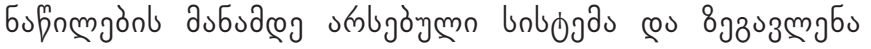

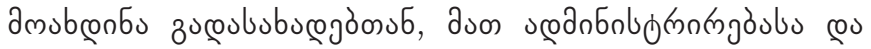

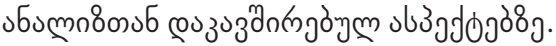

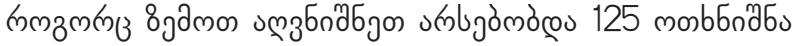

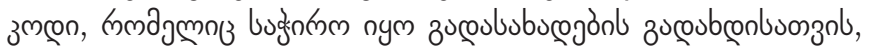

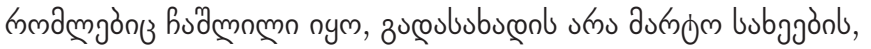

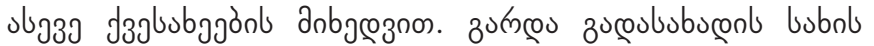

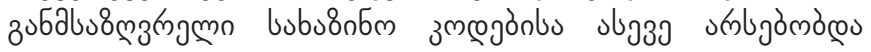

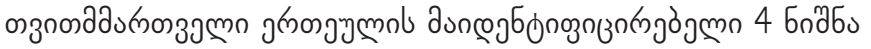

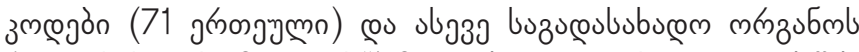

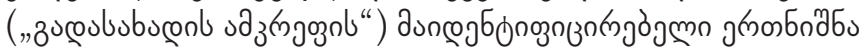

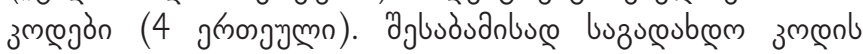

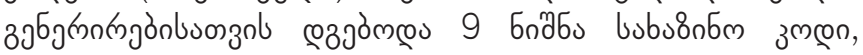

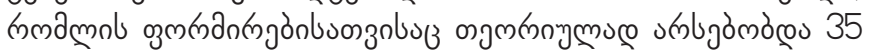

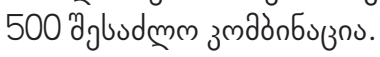

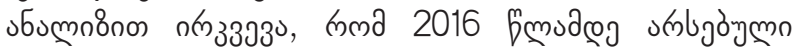

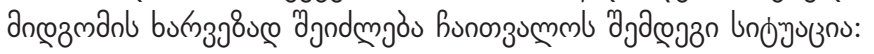

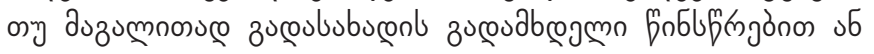

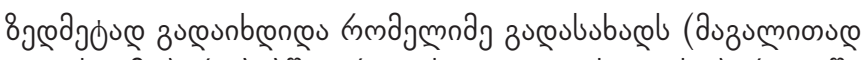

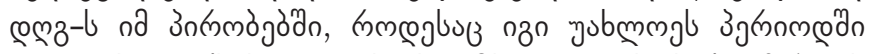
эмmल

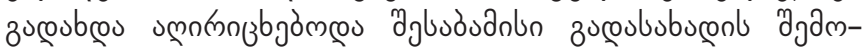

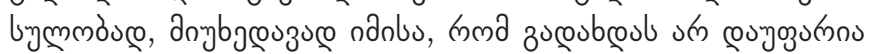

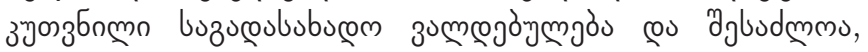

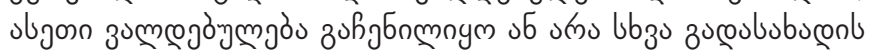
bubgan pou lbas.

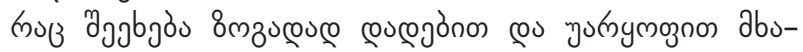

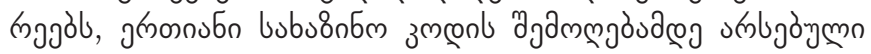

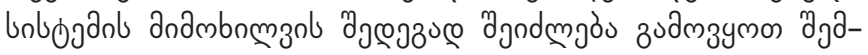

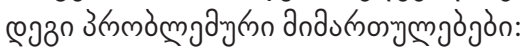

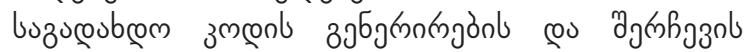

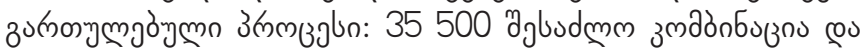

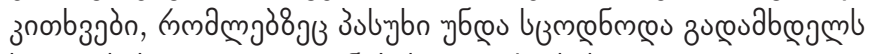

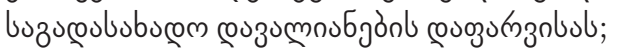

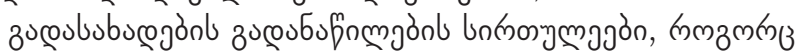

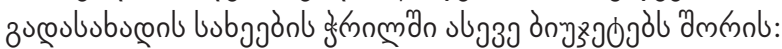

zucoububugonl gmonn bubncoub libju bubgan zucou-

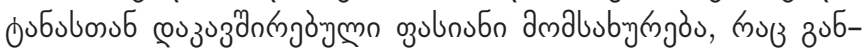

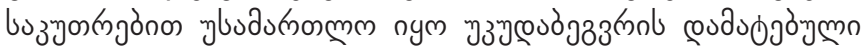

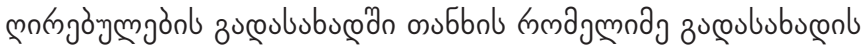

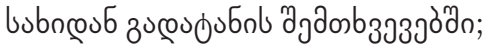

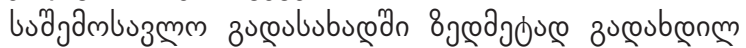

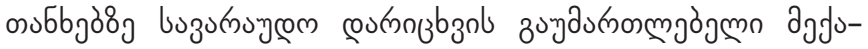

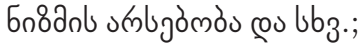

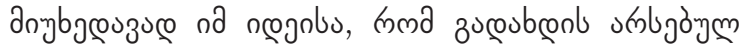

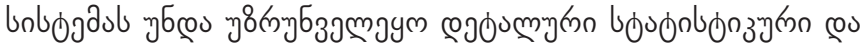

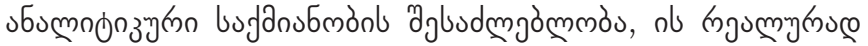
sm dy

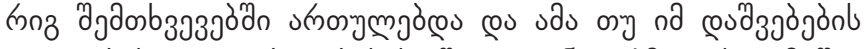

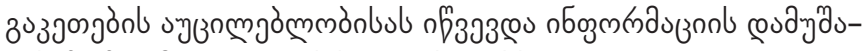

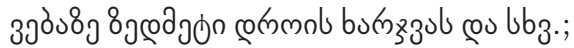

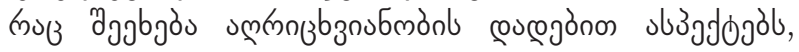

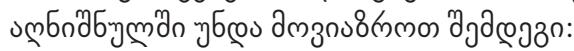

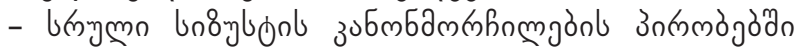

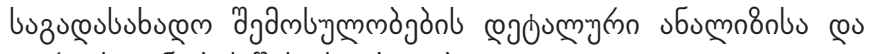

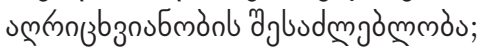

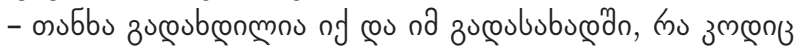

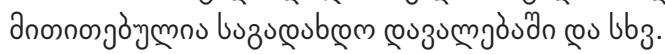

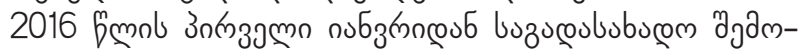

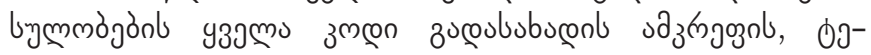

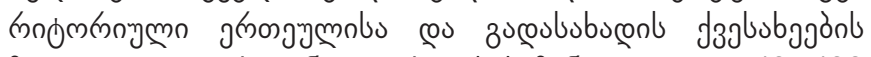

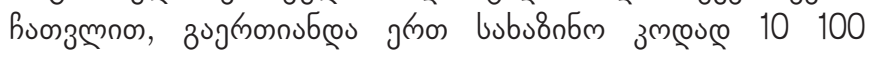




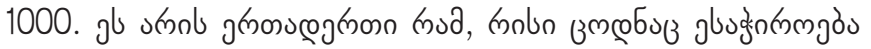

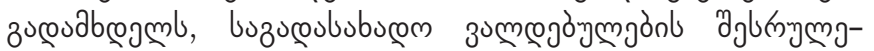

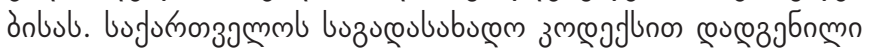

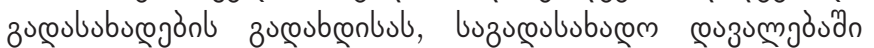

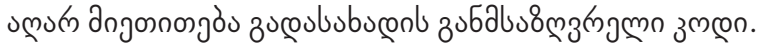

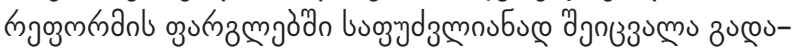

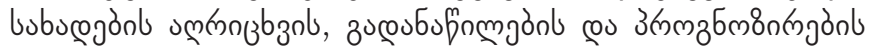

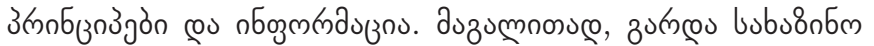

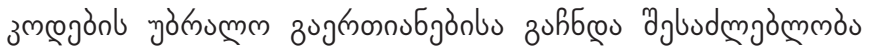

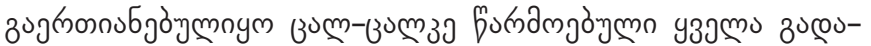

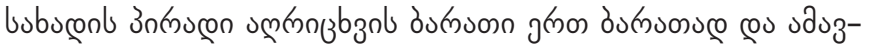

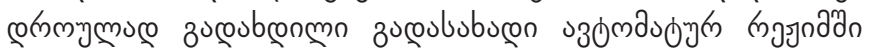

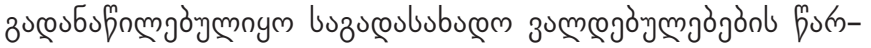

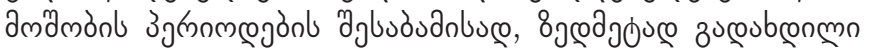

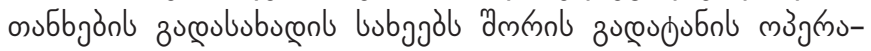
उngönl hooszmnon.

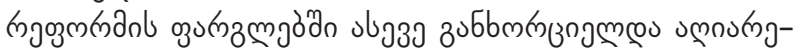

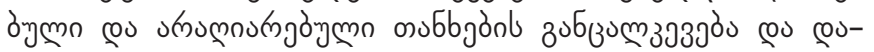

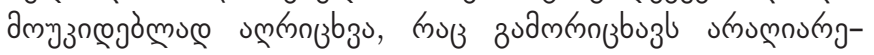

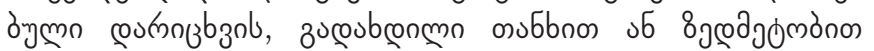

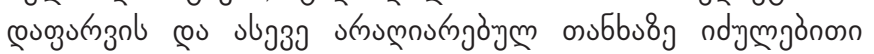

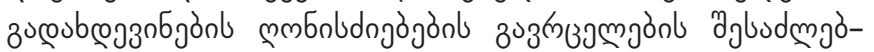

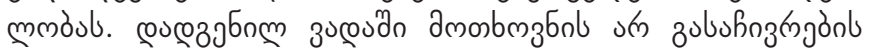

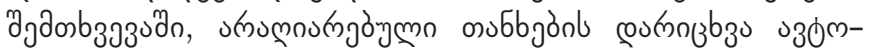

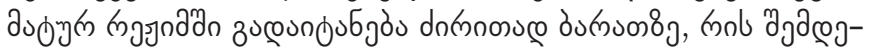

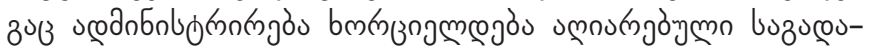

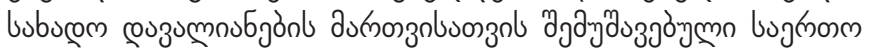

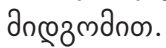

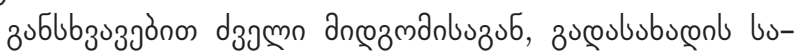

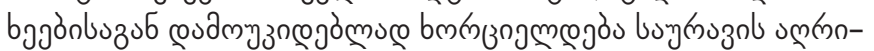

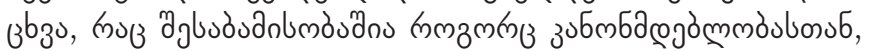

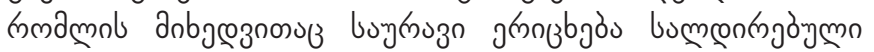

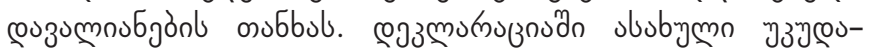

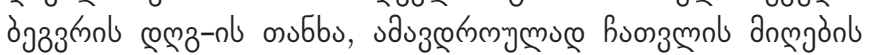
loogydzomno.

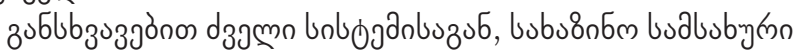

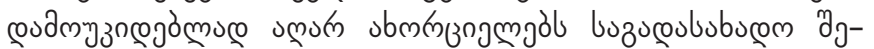

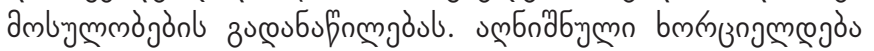

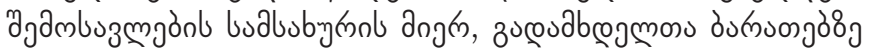

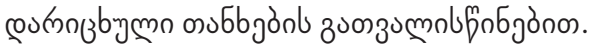

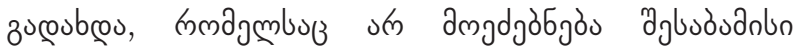

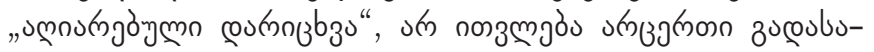

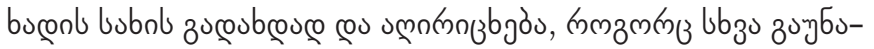

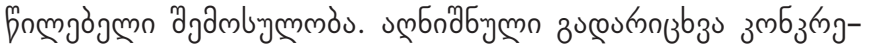

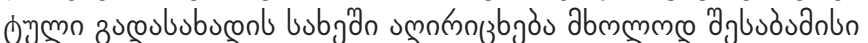

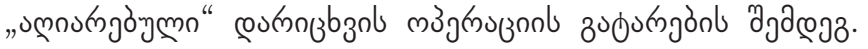

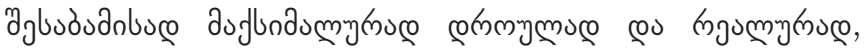

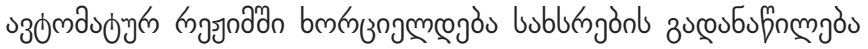

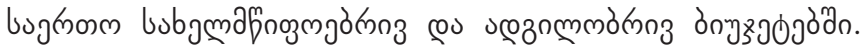

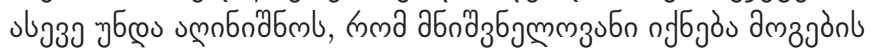

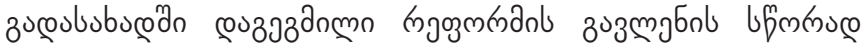

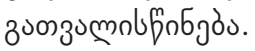

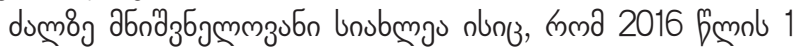

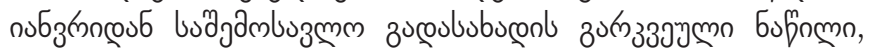

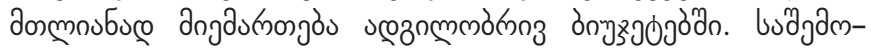

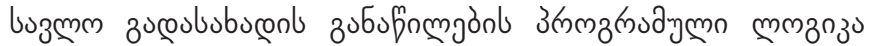

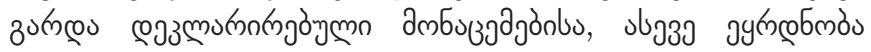

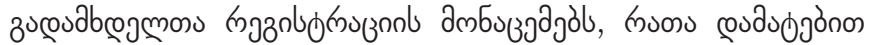

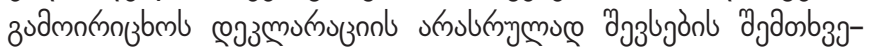

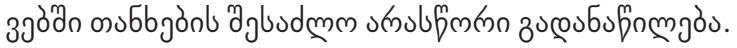

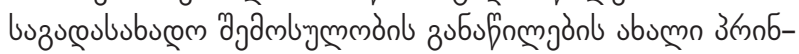

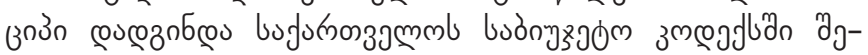

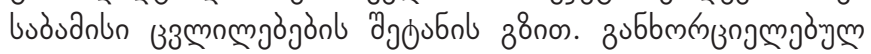

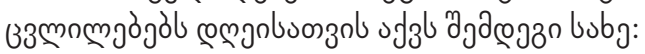

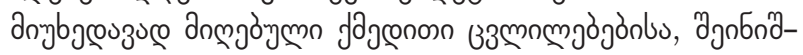

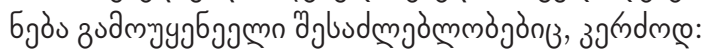

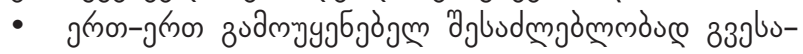

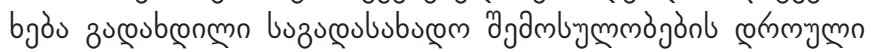

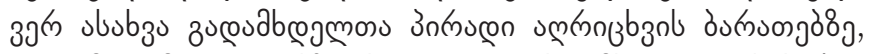

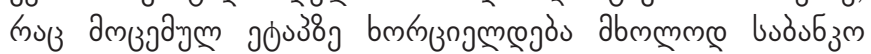

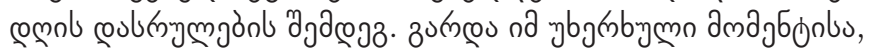

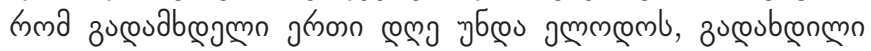

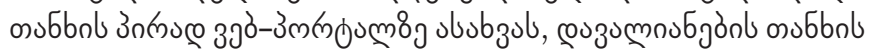

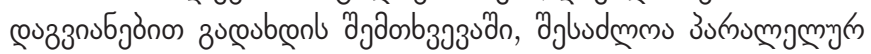

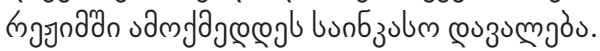

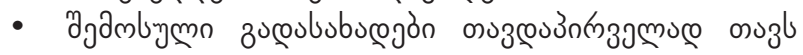

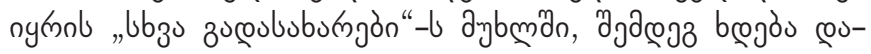

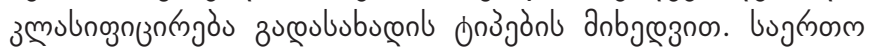

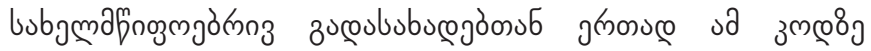

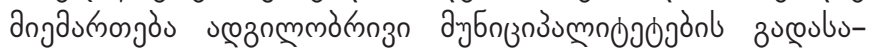

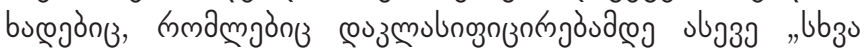

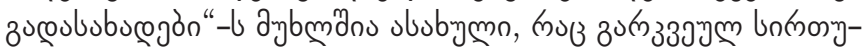

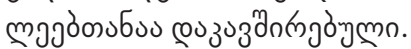

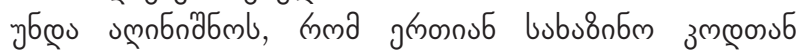

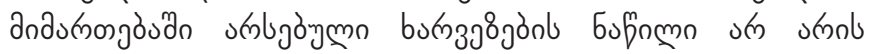

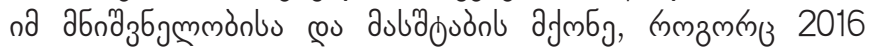

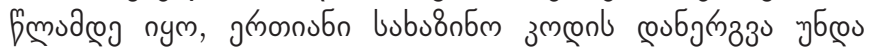

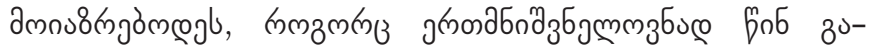

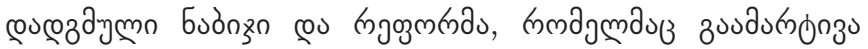

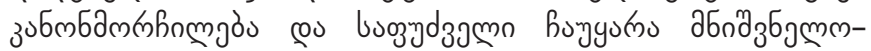

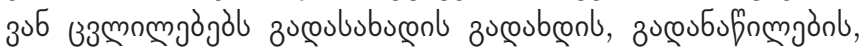

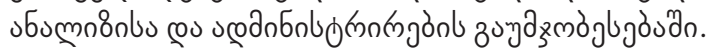




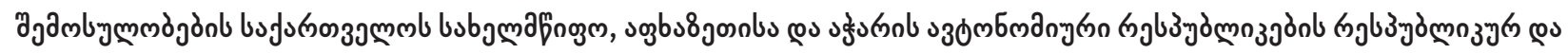

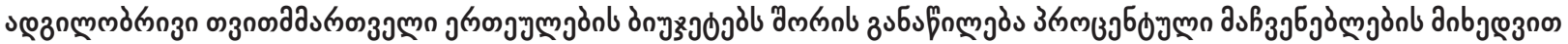

\begin{tabular}{|c|c|c|c|c|}
\hline N & 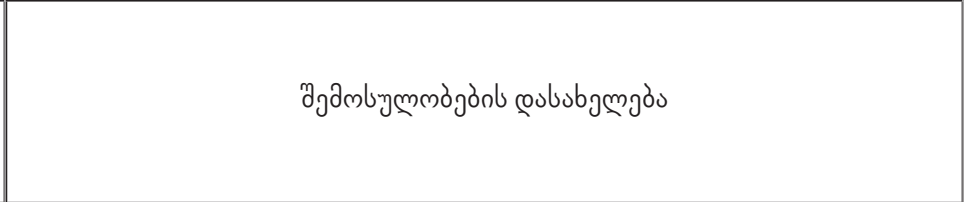 & 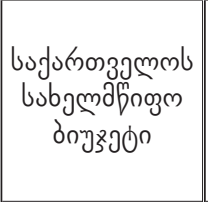 & 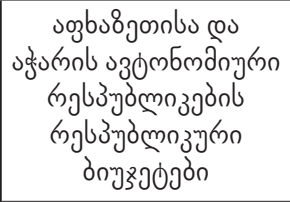 & 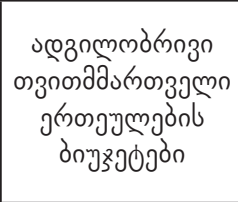 \\
\hline 1 & 2 & 3 & 4 & 5 \\
\hline 1 & buдjдmbuzmm zucoububunn: & & & \\
\hline 1.1 & 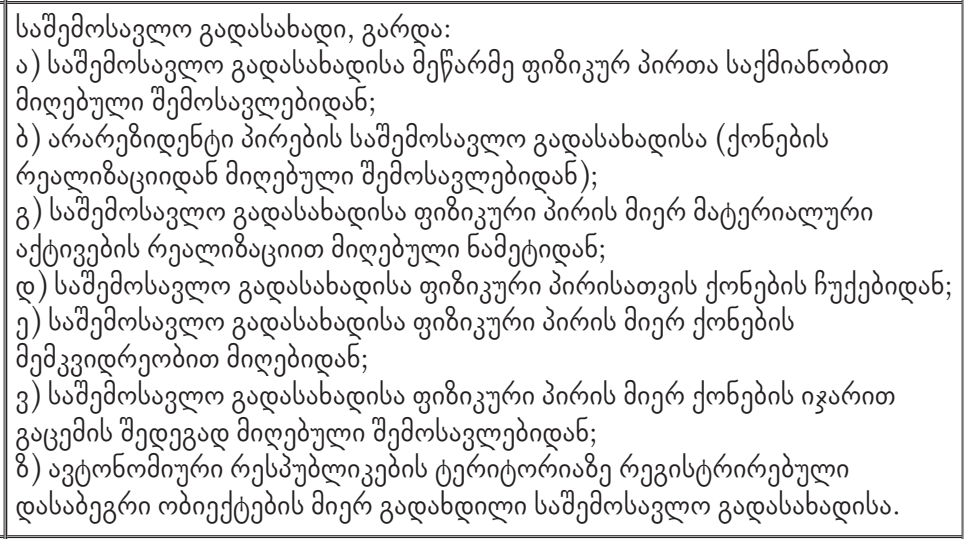 & 100 & & \\
\hline 1.2 & 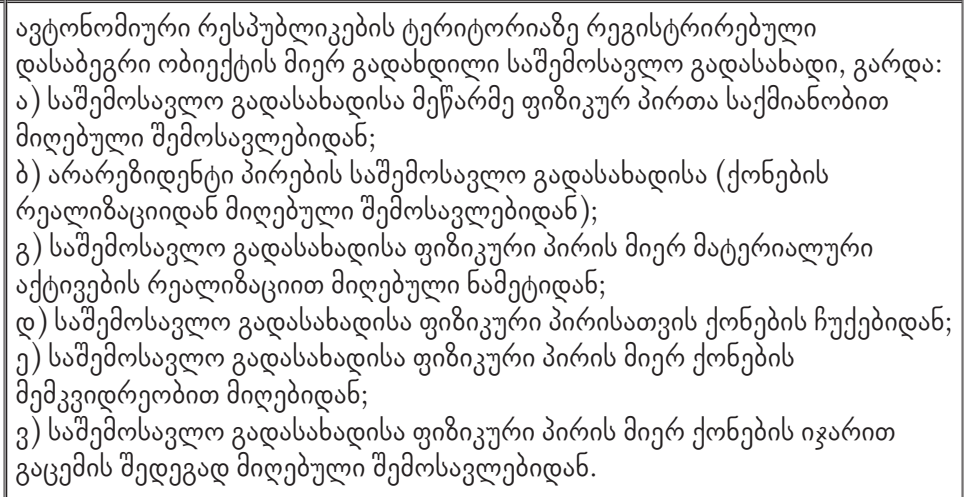 & & 100 & \\
\hline 1.3 & 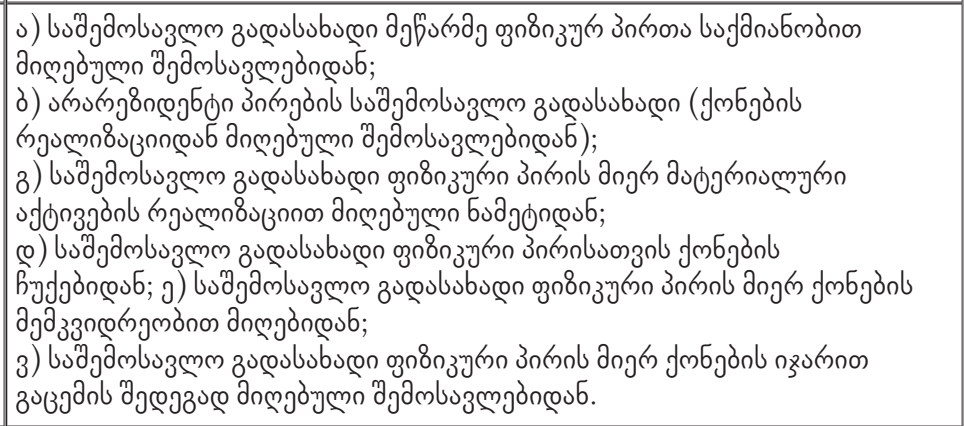 & & & 100 \\
\hline 2 & amzjönl zucoububugn. & 100 & & \\
\hline 3 & jmbjönls zucoububugen. & & & 100 \\
\hline 4 & 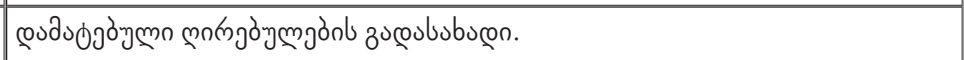 & 100 & & \\
\hline 5 & ofgno̊n. & 100 & & \\
\hline 6 & na3mknonl zucoububuçn. & 100 & & \\
\hline 7 & 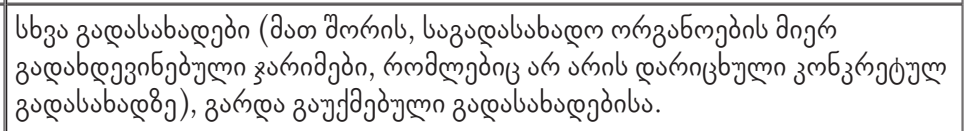 & 100 & & \\
\hline
\end{tabular}

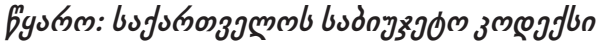




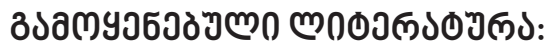

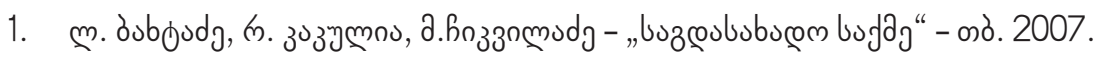

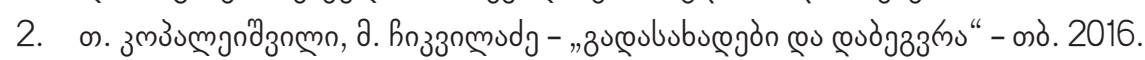

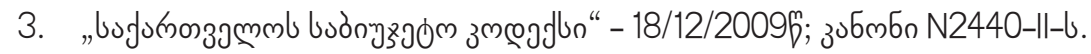

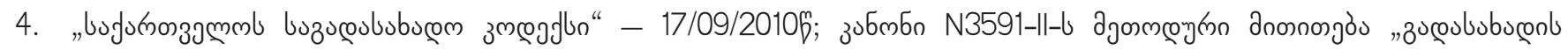

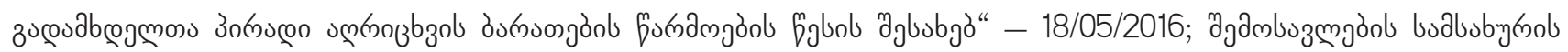
jóndubjò N13446.

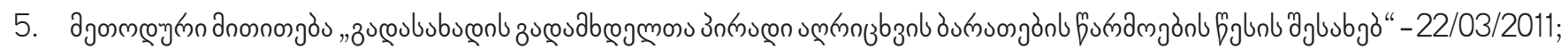

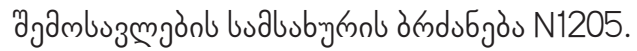

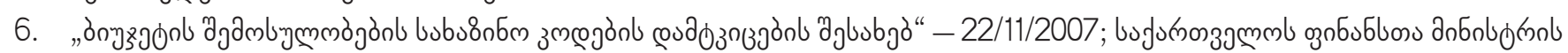
jóndubjos N1226.

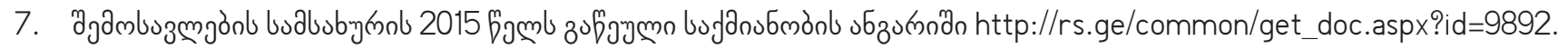




\section{PAYMENT, REDISTRIBUTION AND ANALYSIS OF TAXES AND IMPROVEMENT OF ADMINISTRATION}

\section{MIKHEIL CHIKVILADZE}

https://doi.org/10.35945/gb.2017.04.007

Doctor of Economics, Associate Professor of Ivane Javakhishvili Tbilisi State University, Georgia

KEYWORDS: PAY TAXES, REDISTRIBUTION, ADMINISTRATION

\section{SUMMARY}

There is a very important theme. The main accents there are made on the amendments in the Budget Code in 20162017, namely on the distribution of revenues in the state budget of Georgia and between the autonomous republics of Abkhazia and Adjara from January 1, 2016, according to percentage indicators. There is also important space allocat- ed for analyzing the results of implementation of the Unified Treasury Code since January 1, 2016.

The topics discussed and analyzed in the article allow us to make conclusions on one hand how the factors challenging mobilizing of tax revenues can be mitigated and on the other hand, it shows how to increase the state budget through transparent revenue mechanisms can be stimulated. 